\title{
Supplementary Information S2
}

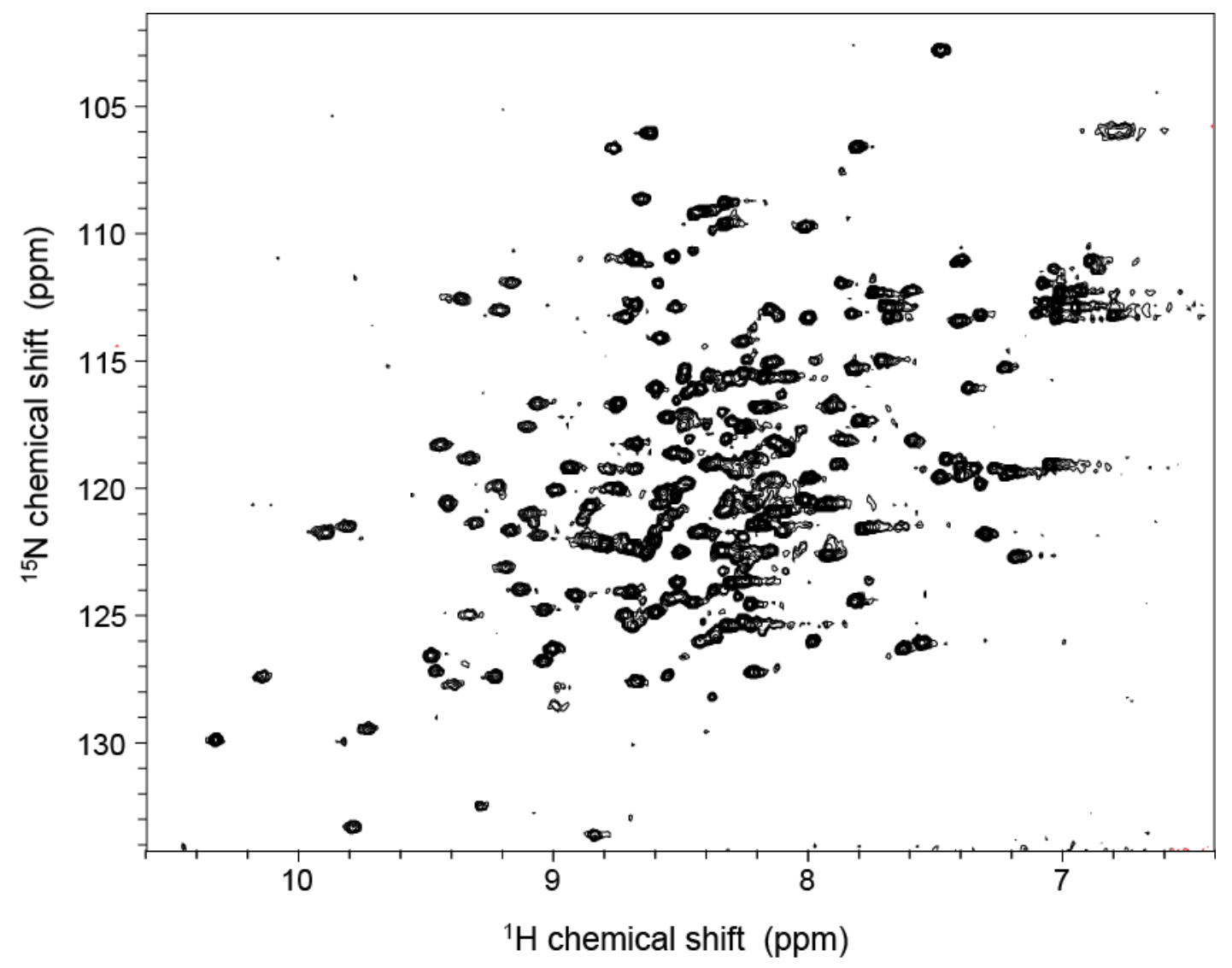

Fig S1. ${ }^{15} \mathbf{N}-H S Q C$ spectrum of $\mathbf{L M O 4}_{\mathbf{1 6}_{16} \mathbf{1 4}} \mathbf{\text { L4}}$-DEAF1. The protein $(115 \mu \mathrm{M})$ was in $20 \mathrm{mM}$ sodium acetate, $35 \mathrm{mM} \mathrm{NaCl}, 1 \mathrm{mM}$ DTT (dithiothreitol) at $\mathrm{pH}$ 5.0. Spectra were recorded on a 600 $\mathrm{MHz}$ spectrometer at $310 \mathrm{~K}$. 

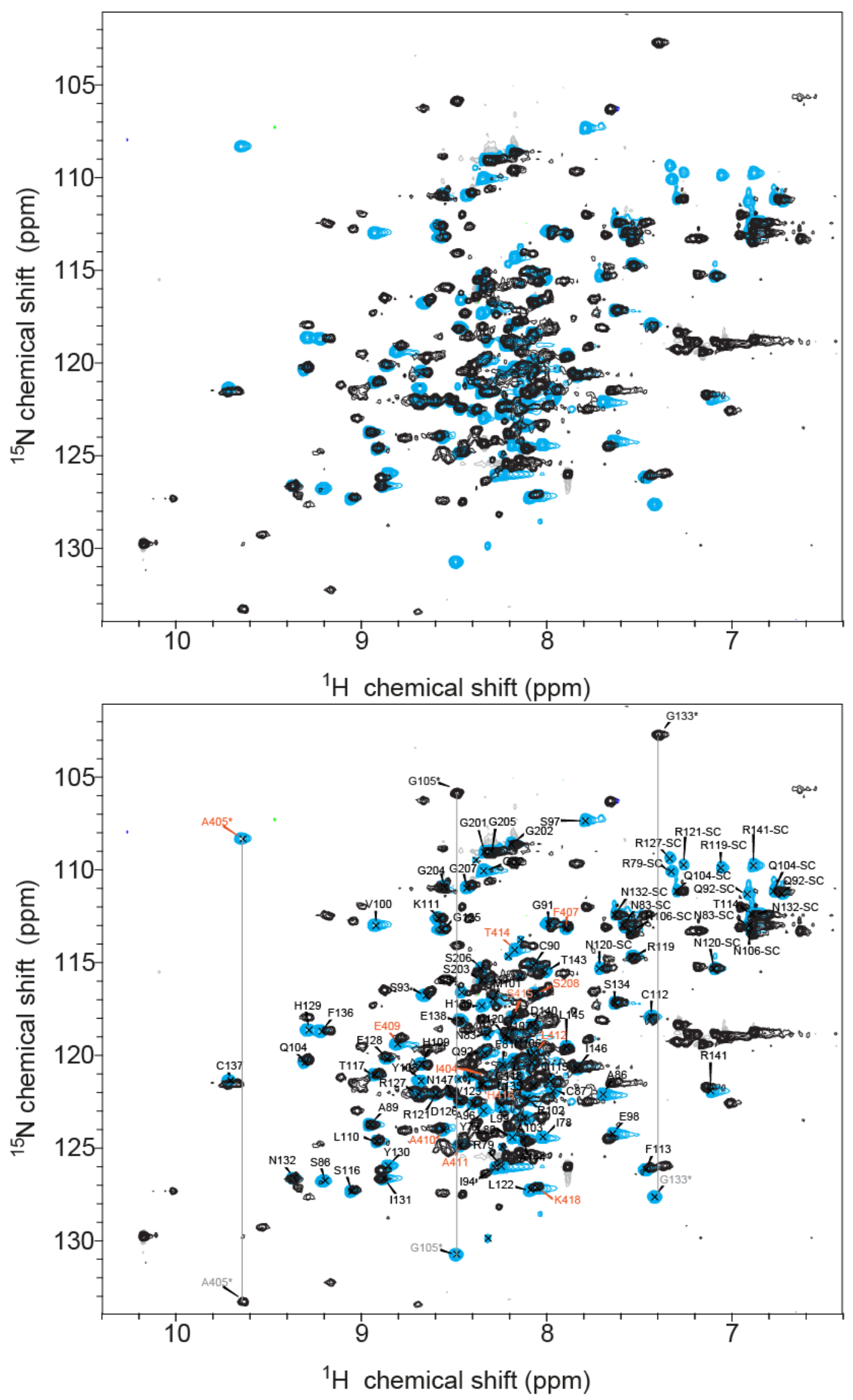

Fig S2. Overlay of ${ }^{15} \mathrm{~N}-\mathrm{HSQC}$ spectra for $\mathrm{LMO4} 4_{\mathrm{LIM1}+2} \cdot \mathrm{L} 4-\mathrm{DEAF1}($ black) and

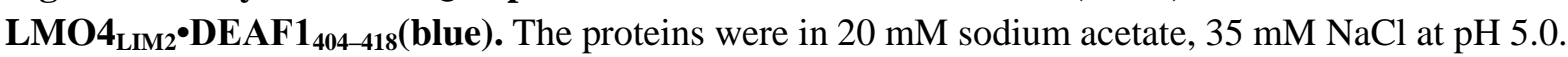


The LMO4 $4_{\text {LIM } 1+2} \cdot$ L4-DEAF1 samples contained $1 \mathrm{mM} \mathrm{DTT}$. The LMO4 $4_{\text {LIM2 }} \cdot \mathrm{DEAF} 1_{404-418} \mathrm{sample}$ also contained TCEP (tris(2-carboxyethyl)phosphine-HCl; $0.5 \mathrm{mM}$ ), chloramphenicol $34 \mu \mathrm{g} / \mathrm{mL}^{-1}$ and Complete EDTA-free protease (Roche; $\sim 0.5 \mathrm{mM}$ ). Spectra were recorded on a $600 \mathrm{MHz}$ spectrometer at $298 \mathrm{~K}$. The top and bottom panels are identical except that the bottom panel includes labels showing peak assignments for $\mathrm{LMO}_{\text {LIM2 }} \cdot \mathrm{DEAF}_{404-418}$ [1] (BMRB: 18898). Labels for S208 from the linker and DEAF1 are in orange. The peaks marked with an asterisks $(*)$ were aliased for $\mathrm{LMO}_{\mathrm{LIM}_{2}} \cdot \mathrm{DEAF} 1_{404-418}$ and the likely corresponding peaks in LMO4 $4_{\text {LIM1+2 }} \cdot$ L4-DEAF1 are indicted (grey lines and labels).

There is substantial overlap of the spectrum of the smaller complex with that of the larger complex. Although the peaks for $\mathrm{LMO} 4_{\mathrm{LIM} 1+2} \cdot \mathrm{L} 4$-DEAF1 have not been assigned, by inference peaks corresponding to residues from S208 and DEAF $1_{404-411}$, and most residues in the $\mathrm{LMO}_{\text {LIM2 }}$ domain from $\mathrm{LMO} 4_{\text {LIM2 }} \cdot \mathrm{DEAF} 1_{404-418}$ are recapitulated in the spectrum of the larger construct. Peaks corresponding to $\mathrm{DEAF}_{412-418}$ are either in crowded regions (making them impossible to distinguish) or appear to have shifted. 


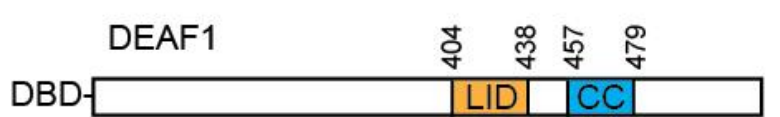

wt

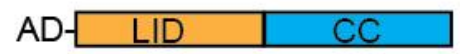

434-36

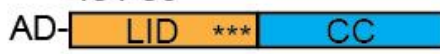

LID- LMO4 interaction domain

CC - Coiled-coil domain

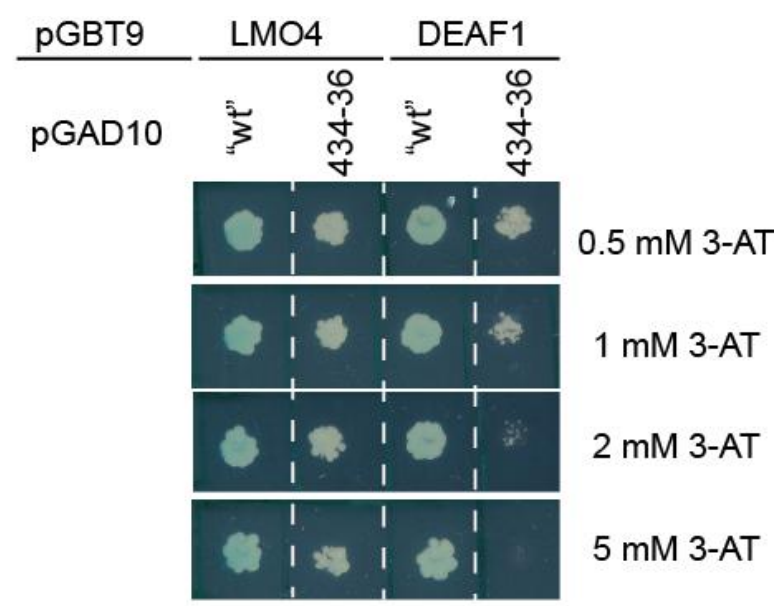

Fig S3. The 434-36 triple alanine mutant of DEAF1 is destabilised in yeast compared to wildtype. Yeast-two hybrid assays were used to compare the interaction of wt and mutant pGAD10DEAF1 $1_{404-438 \_457-479}$ with pGBT9-LMO4 and pGBT9-DEAF1 45-566, via the LMO4 interaction domain and coiled-coil domain of DEAF1, respectively. The 434-36 triple alanine mutant construct shows significant loss of self interaction through the coiled-coil domain under selection conditions that allow interaction with LMO4 (-L-W-H plus 0.5-5 mM 3-AT), suggesting that the mutant construct is destabilised and/or degraded compared to the wild-type ("wt"). Note that self interaction of DEAF1 via the coiled-coil domain was reported previously [2], and no growth of the mutant with LMO4 is seen under high stringency selection conditions (-L-W-A; Fig 1c).

\section{References}

[1] Joseph, S., Kwan, A. Y., Mackay, J., Cubeddu, L. \& Matthews, J. M. (2014). Backbone and side-chain assignments of a tethered complex between LMO4 and DEAF-1. Biomol NMR Assign $\mathbf{8}$, $141-4$.

[2] Cubeddu L, Joseph S, Richard DJ, Matthews JM (2012) Contribution of DEAF1 structural domains to the interaction with the breast cancer oncogene LMO4. PLoS ONE 7: e39218. 